\title{
Development of a Specific Latex Agglutination Test to Detect Antibodies of Enterovirus 71
}

\author{
Liu $\mathbf{X}^{1^{*}}$, Qin $\mathbf{B}^{1,2,3}$, He $\mathrm{T}^{2}$, Chen $\mathrm{J}^{2}$, Cao $\mathrm{D}^{2}$ and Xie $\mathbf{W}^{4}$ \\ ${ }^{1}$ Medical College of Shaoxing University, Shaoxing, China \\ ${ }^{2}$ Shaoxing Centre for Disease Control and Prevention, Shaoxing, Zhejiang Province, China \\ ${ }^{3}$ Molecular Medicine Center of Shaoxing People's Hospital, Shaoxing Hospital of Zhejiang University, Shaoxing, China \\ ${ }^{4}$ Institute for Applied Nephrology (Interneph), Fuchserde 7, Aachen, Germany
}

\begin{abstract}
A latex agglutination test (LAT) was developed for the rapid detection of antibodies against the VP1 or VP1 proteins of Enterovirus 71 (EV71). The proteins of interest, including prokaryotically expressed VP1 and two strains of anti-VP1 Monoclonal antibody (McAb) against EV71 were covalently linked to carboxylated latex using Ethyl-dimethyl-aminopropyl carbodiimide (EDC) to prepare sensitized latex beads. The LAT was evaluated by an enzyme-linked immunosorbent assay (ELISA) as a reference test. The VP1-LAT showed a sensitivity of $87.0 \%$, a specificity of $88.9 \%$, and an agreement ratio of $90.0 \%$ in detecting VP1 in 100 serum samples from experimentally infected mice, while these values were $86.8 \%, 96.7 \%$, and $93.3 \%$, respectively, for 608 clinical human serum samples. The VP1-LAT has advantages over other assays in terms of low cost, rapidity, chemical stability, high sensitivity, repeatability, and specificity. The LAT established in the present study is a rapid and simple test suitable for field monitoring of antibodies against VP1-EV71.
\end{abstract}

Keywords: Enterovirus 71; Hand-foot-and-mouth disease; Latex agglutination test; Homogeneity; Ethyl-dimethyl-amino-propyl carbodiimide

\section{Introduction}

Hand, foot and mouth disease (HFMD) is a common childhood disease that is characterized by fever, a rash on the hands and feet, and mouth ulcers $[1,2]$. Most children, usually under five years old, with HFMD carry Coxsackievirus A and/or Enterovirus 71 (EV71) [3]. It is usually a mild and self-limiting febrile disease in children, however, EV71 infection has been associated with various neurological diseases, such as aseptic meningitis, polio-like paralysis, and acute encephalitis with neurological pulmonary edema, mainly in young children and infants $[1,4,5]$.

The neurovirulence of EV71 first drew public concern in 1975 in Bulgaria when 44 people died of a polio-like disease [6]. EV71 was correlated with epidemics of neuroinvasive diseases in New York, Malaysia, Japan, Australia, Taiwan, China, Hungary, and Hong Kong [7]; thus, it is regarded as an emerging neurotropic enterovirus, as is poliovirus $[6,8]$.

EV71 is a non-enveloped virus, belonging to the genus Enterovirus of the Picornaviridae family, with a positive, single-stranded positivepolarity RNA of about $7.5 \mathrm{~kb}$ in size $[9,10]$,with single Open reading frame (ORF) encodes a huge polyprotein of 2,194 amino acids and is flanked by a 5'-untranslated region (UTR) and 3'-UTR, which can be divided into three regions: $\mathrm{P} 1$ consisting of the four structural proteins VP1 to VP4, and P2 and P3 [11].

For seroepidemiological surveys and evaluations of vaccine effects, EV71 IgG from serum samples needs to be detected. At present, the serologic test for EV71 is the Enzyme-linked immunosorbent assay (ELISA), which takes a minimum of several hours and requires trained personnel and special equipment $[12,13]$. In our study, we isolated epidemic strains of EV71 from clinical specimens. After being identified by reverse transcribed PCR (RT-PCR) [14,15], the VP1 was amplify and the protein was expressed [16]. For the detection of IgG against EV71 VP1, we used prokaryotically expressed VP1 as bait to establish the latex agglutination method to detect antibodies against EV71. After optimizing the experimental parameters, the latex agglutination method could specifically detect the relevant target. The anti-VP1 IgG detection method was not dependent upon a secondary antibody, such as in an ELISA or immunofluorescence, and it can detect IgG from all kinds of serum samples.

Latex agglutination, among all antigen detection assays, is the most rapid and simplest to perform $[17,18]$. In this study, we describe a simple and specific Latex agglutination test (LAT) that is based on the VP1 protein for the rapid detection of VP1-specific antibodies in sera. The specificity and sensitivity of the assay was compared with a commercial ELISA kit. LAT is a specific, sensitive, accurate, fast and simple IgG-detection method which has potential value for field applications.

\section{Materials and Methods}

\section{EV71 isolation from clinical samples}

Throat swabs anal swab samples of patients diagnosed with HFMD were collected, and were sorted into EV71 or coxsackievirus A16 (Cox A16) by real-time PCR. Swab samples identified as EV71-positive were filtered using a $0.22 \mu \mathrm{m}$ filter (Millipore, Billerica, MA, USA) and added to the wells of Vero cells cultured in Dulbecco's modified Eagle's medium (DMEM; Invitrogen, Carlsbad, CA, USA) at $37^{\circ} \mathrm{C}$ in a $5 \% \mathrm{CO}_{2}$ atmosphere supplemented with $10 \%$ fetal bovine serum (Gibco, Grand Island, NY, USA), $2 \mathrm{mM}$ of glutamine, $100 \mathrm{IU} / \mathrm{ml}$ of penicillin, and $100 \mathrm{IU} / \mathrm{ml}$ of streptomycin. The supernatant was collected and stored at $-80^{\circ} \mathrm{C}$ until the cytopathic effect $(\mathrm{CPE})$ took place, regardless of whether it was the first or second passage. Over time, the growth of the

*Corresponding author: Liu X, Department of Histology and Embryology, Medical College of Shaoxing University, Shaoxing, The People's Republic of China, Tel: 860575-88137362; Fax: 86-0575-88137362; E-mail: 13967506627@126.com

Received: March 14, 2015; Accepted: March 30, 2015; Published: April 04, 2015

Citation: Liu X,Qin B, He T,Chen J,Cao D, et al.(2015) Development of a Specific Latex Agglutination Test to Detect Antibodies of Enterovirus 71. Metabolomics 5: 142. doi:10.4172/2153-0769.1000142

Copyright: (c) 2015 Qin B, et al. This is an open-access article distributed under the terms of the Creative Commons Attribution License, which permits unrestricted use, distribution, and reproduction in any medium, provided the original author and source are credited. 
Citation: Liu X,Qin B, He T,Chen J,Cao D, et al.(2015) Development of a Specific Latex Agglutination Test to Detect Antibodies of Enterovirus 71. Metabolomics 5: 142. doi:10.4172/2153-0769.1000142

Page 2 of 7

vero cells gradually declined. CPE appeared as early as 72 hours, and as late as the second passage. Two samples resulted with typical CPE are shown in Figures $1 \mathrm{~A}$ and $1 \mathrm{~B}$.

To clarify the sequence and genotype of the local epidemic EV71 strain, 10 EV71 strains were isolated from clinical samples of HFMD children [19]. Positive samples identified by RT-PCR were added to the wells seeded with Vero cells.

\section{Genotype analysis of isolated EV71}

The cDNA of EV71 was acquired by reverse transcription using the extracted RNA from the culture supernatant as template and an oligo (dT) primer. Then, the sequence of VP1 was amplified by PCR using pEV71-F and - R primers (Table 1) and then subjected to sequencing. Both the nucleotide and amino acid sequences of VP1 of isolated EV71 strains were compared with that of $\mathrm{A}, \mathrm{B}$, and $\mathrm{C}$ genotype reference EV7l strains [20].

\section{Production and purification of recombinant VP1 protein}

The production of recombinant VP1 was conducted as follows. After EV71 RNA was extracted, cDNA was acquired by reverse transcription and used as template to amplify the VP1 with the pEV71-F and $-\mathrm{R}$ primers (Table 1), which contained the unique restriction sites BamHI and HindIII at the 5' and 3' ends, respectively. A 1,015-bp PCR product was detected by electrophoresis and then recovered, digested with BamHI and HindIII, and ligated into the same sites in pET28(a)+ using T4 DNA ligase. The resulting vector, pET-28a-VP1, in which VP1 is fused at the C-terminal end of the Glutathione-S-transferase (GST) protein, was transformed into E. coli strain BL21, and GSTVP1 expression was induced by adding isopropyl-b-D-thiogalactoside (IPTG). Proteins were purified on a GST-affinity column, and dialyzed in Phosphate-buffered saline (PBS) for three days in the next step [18]. The purity of the proteins was analyzed by Sodium dodecyl sulfate- polyacrylamide gel electrophoresis (SDS-PAGE) and confirmed by Western blot analysis with mouse sera against the VP1 or EV71 [21].

\section{Serum samples}

Serum samples in our study include mouse serum, rabbit serum, and human serum (adult and infant). Mice and rabbits were challenged with live/inactive isolated EV71 viruses or expressed VP1 protein to acquire different positive sera. 400 serum samples from 3 to 5-yearold children were collected, some of whom were previously infected with HFMD. 100 serum samples from adults were also collected for detection.

\section{Preparation and optimization of sensitized latex beads}

Sensitized latex beads were prepared as follows. In brief, $0.7 \mu \mathrm{m}$ radius $10 \%$ latex beads (Ke Xin Company, Shanghai, China) were bridged with 1-ethyl-3-(3-dimethylaminopropyl) carbodiimide (EDC) and then conjugated with different concentrations of VP1 protein. The beads were washed gently three times with $0.1 \mathrm{M}$ carbonate buffer ( $\mathrm{pH}$ 9.6) and then three times with $0.02 \mathrm{M}$ phosphate buffer ( $\mathrm{pH} 4.5$ ), then suspended in 2\% EDC solution and 2-hours-incubated on a shaker at $37^{\circ} \mathrm{C}$. After washing three times with boric acid buffer ( $\mathrm{pH}$ 8.4), VP1 was added and rocked gently for $6 \mathrm{~h}$. Finally, the bead pellet was suspended in latex storage buffer (1\% BSA, 5\% glycerol, $0.1 \%$ $\mathrm{NaN}_{3}$ in PBS, pH 7.4) and stored at $4^{\circ} \mathrm{C}$ prior to use $[17,18,22]$. For the best sensitivity and specificity of the LAT, a series of experiments were conducted to identify the optimal concentrations for protein and latex beads, as well as the length of the conjugation reaction. $100 \mu \mathrm{l}$ of VP1 (500 ng/ $\mu$ l) (serial two-fold dilutions from 1:1 to 1:16) was used to sensitize $200 \mu \mathrm{l}$ of latex beads $(1 \%)[18,23]$. The primed beads were mixed with PBS or positive serum and observed for autoagglutination or agglutination. The optimum concentration was defined as the most sensitive and economical concentration that did not result in autoagglutination. Determination of the optimal reaction time for
$24 \mathrm{~h}$

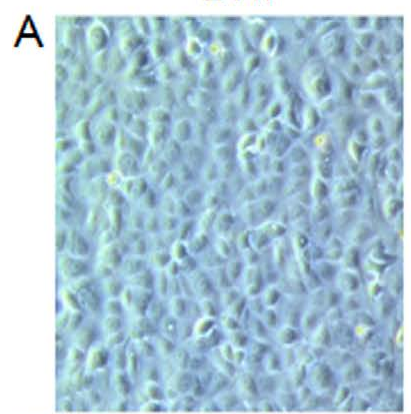

B

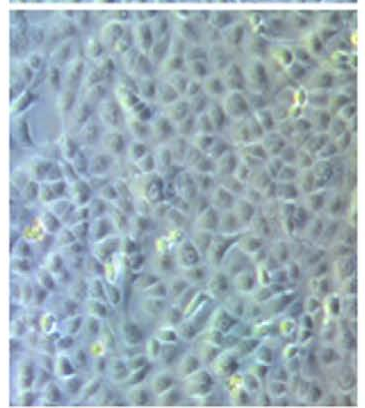

$48 \mathrm{~h}$
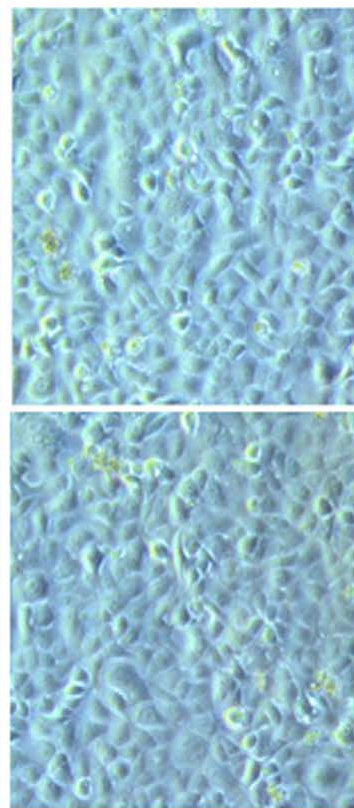

$72 \mathrm{~h}$
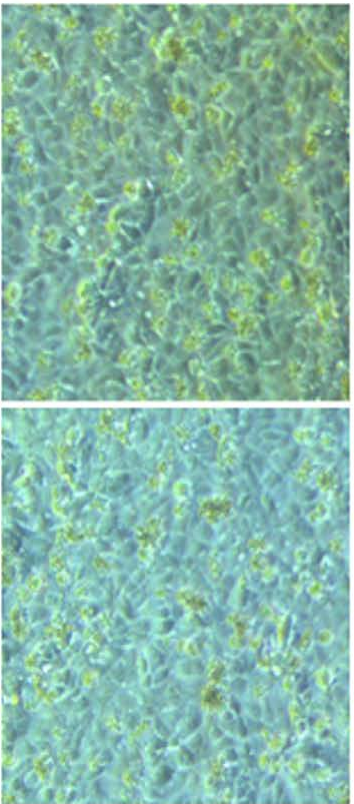

$96 \mathrm{~h}$
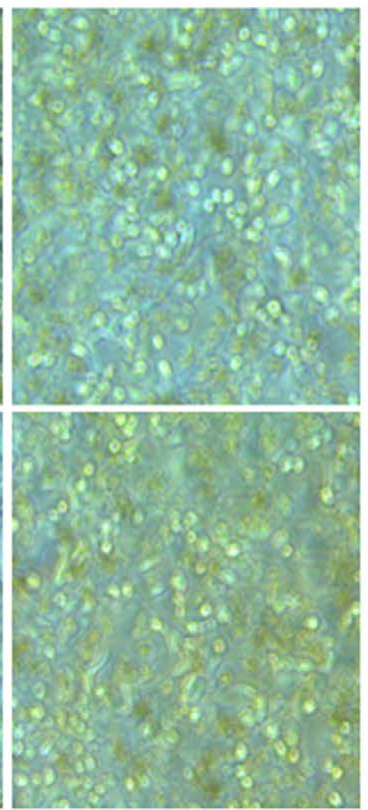

Figure 1: EV71 isolated form clinical specimens using Vero cells. EV71-positive samples identified by RT-PCR were used to inoculate Vero cells. Two specimens (A and $B$ ), which brought about typical cytopathic effects (CPE) at 24, 48, 72 , and 96 hours, were chosen to be tested. 


\begin{tabular}{|c|c|c|c|}
\hline Name & Location & Size (bp) & Sequence \\
\hline & $446-600$ & 154 & 5'-TCCTCCGGCCССTCAATGCG-3' \\
\hline $\mathrm{pEV}-\mathrm{R}$ & & & 5'-ATTGTCACCATAAGCAGCCA-3' \\
\hline $\mathrm{pEV} 71-\mathrm{F}$ & 2372-2392 & 226 & 5'-GCAGCCCAAAAGAACTTCAC-3' \\
\hline $\mathrm{pEV} 71-\mathrm{R}$ & 2578-2598 & & 5'-ATTTCAGCAGCTTGGAGTGC-3' \\
\hline pCA16-F & $2335-2355$ & 208 & 5'-ATTGGTGCTCCCACTACAGC-3' \\
\hline pCA16-R & $2523-2543$ & & 5'-TCAGTGTTGGCAGCTGTAGG-3' \\
\hline pEV71-VP1-F & 2367-2282 & 1015 & 5'-CGGGATCCGGAGATAGGGTGGCAGATGTA-3' \\
\hline pEV71-VP1-R & & & 5'-CGGAATCCAGTAGTGATCGCCGTGCG-3' \\
\hline
\end{tabular}

Primer-EV-forward coupled with primer-EV-reverse were designed as universal primers of enterovirus, and used to amplify a 154-bp segment shared by enteroviruses, including EV71, EV70, coxsackie virus A, and poliovirus. Primer-EV71-forward and -reverse were designed to detect EV71, while pCA16-forward and -reverse were for CoxA16 detection. Primer EV71-VP1-forward and -reverse were used to construct pET28a-VP1.

Table 1: Primer sequences for pET28a-VP1 construction and qRT-PCR.

the sensitization and concentration of the latex beads was performed similarly in a systematic fashion [18].

\section{Latex agglutination test}

The LAT was performed by mixing $15 \mu$ samples with $15 \mu \mathrm{l}$ prepared beads on a microscope slide with a plastic tip or toothpick, the slide was rocked manually for about 1 to 2 minutes which depend on temperature, rocking amplitude and IgG titer [18]. Test results were scored as follows: " ++++ ", rapid agglutination of $100 \%$ of the sensitized latex beads; " +++ ", $75 \%$ sensitized latex beads agglutinated; "++", agglutination of $50 \%$ of the sensitized latex beads; "+", fine particle agglutinated, about $25 \%$ of the sensitized latex beads with a questionable interpretation; “-”, no visible agglutination greater than negative control. Agglutination reactions "+" to " ++++ " were considered to be positive results.

\section{Results}

\section{Construction of pET-28(a)-VP1 and expression of VP1}

As described above, VP1 was PCR-amplified, and then pET-28aVP1 was constructed successfully. Then VP1 protein was expressed in E. coli strain BL21 and purified on a GST-affinity column and then dialyzed in PBS for three days. The purity of the protein was analyzed by SDS-PAGE $[13,18]$. As shown in Figure $2 \mathrm{~A}$, VP1, which has a molecular weight of $37 \mathrm{kDa}$, was successfully expressed. Purified VP1 was identified by Western blot analysis with sera from mice challenged with EV71 (Figure 2B, lane 1), sera from mice immunized with VP1 (Figure 2B, lane 2), and a commercially available VP1 polyclonal antibody (Figure 2B, lane 3). It turned out that expressed VP1 protein in our study keeps approving immunogenicity and reactogenicity.

\section{Optimization of latex bead sensitization}

Different antigen concentrations were used to sensitize 10\% latex beads with an overnight reaction time (as the starting condition, as recommended by the latex bead manufacturer). A standard positive serum sample was serially diluted to react with the sensitized latex beads; the final serum dilution with positive agglutination was recorded as the serum titer [18].

Samples containing the VP1 protein were prepared at an initial concentration of $500 \mathrm{ng} / \mu \mathrm{l}$, and then serially diluted two-fold, from 1:1 to $1: 64$. Next, $200 \mu \mathrm{l}$ of latex beads were washed as previously described [18]. Serially diluted $100 \mu \mathrm{l}$ samples containing the VP1 protein were added to the EDC-processed latex beads solution. After 6 hours of gentle rocking, sensitized latex beads were prepared. As shown in Table 2 , the most appropriate dilution that did not exhibit autoagglutination was economical, and which had the highest titer for positive sera were the 1:8 dilution for the VP1 protein. That is to say that $6,250 \mathrm{ng}$ of VP1 protein per $200 \mu \mathrm{l}$ of latex beads stored in $750 \mu \mathrm{l}$ of buffer could be used to screen 500 samples.

To determine the optimal sensitization time, $100 \mu \mathrm{l}$ of VP1 in a $1: 8$ dilution of the original concentration $(500 \mathrm{ng} / \mu \mathrm{l})$ was placed into a solution of EDC-processed latex beads. The optimal time for the conjugation reaction was reported as 5 hours in our previous study $[18,23]$. However, as shown in Table 3 , from 2 to 6 hours, there was a time-dependent increase in sensitivity, while 7 and 8 hours of conjugation did not further increase the sensitivity (Table 3). Thus, 6 hours was adopted in the VP1-LAT due to a slightly higher sensibility, timeliness, and lack of autoagglutination [18].

VP1-conjugated latex beads react well with anti-VP1 in sera. The LAT was performed by mixing $15 \mu \mathrm{l}$ of positive serum with 15 $\mu \mathrm{l}$ of sensitized latex beads on a microscope slide with a plastic tip or toothpick, and then the slide was rocked manually for 30-60 second $[18,23]$. According to the results shown in Figure 3, VP1-sensitized latex beads can react with the sera of EV71-infected patients (Figure 3B), rabbits (Figure 3C), and mice (Figure 3D) quickly and thoroughly compared with the negative control (Figure $3 \mathrm{~A}$ ). A positive reaction demonstrated a mixture of clear solution and macroscopic particles, while the negative control exhibited a uniform emulsion. We also selected 8 samples of patients to do LAT, the results shown in Fig.3E-L ranged from negative (-) to positive (+ to ++++ ).

\section{Specificity, repeatability, and stability of VP1-LAT}

To examine the specificity of the latex beads, standard sera positive for a wide range of enteroviruses (enteric cytopathogenic human orphan virus, coxsackievirus A16, norovirus, poliovirus, EV70, and hepatitis A virus), and these virus strains were collected and verified using a commercial ELISA kit and real-time PCR [24]. Six lots of LAT (both VP1 and anti-VP1) were involved in this assay, and used to detect these standard positive sera. These standard sera did not react with the EV71-VP1-LAT, except for the EV71-positive serum

(Table 4), indicating that EV71-VP1-LAT established in the present assay was specific for EV71 only.

These six lots of the LAT were also used to test 50 serum samples, all detections were repeated three times with the same batch of beads. The stability of the sensitized latex was judged by performing the VP1LAT on 50 serum samples three times with the same batch of beads: on the day of preparation, and after 1.5, 3, 4.5, and 6 months of storage at $2-8^{\circ} \mathrm{C}$ in a refrigerator [23]. Assuringly, all of the repeated tests yielded the same results (data not shown). Thus far, the LAT shows high sensitivity, satisfactory repeatability, and stability. 
Citation: Liu X,Qin B, He T,Chen J,Cao D, et al.(2015) Development of a Specific Latex Agglutination Test to Detect Antibodies of Enterovirus 71. Metabolomics 5: 142. doi:10.4172/2153-0769.1000142

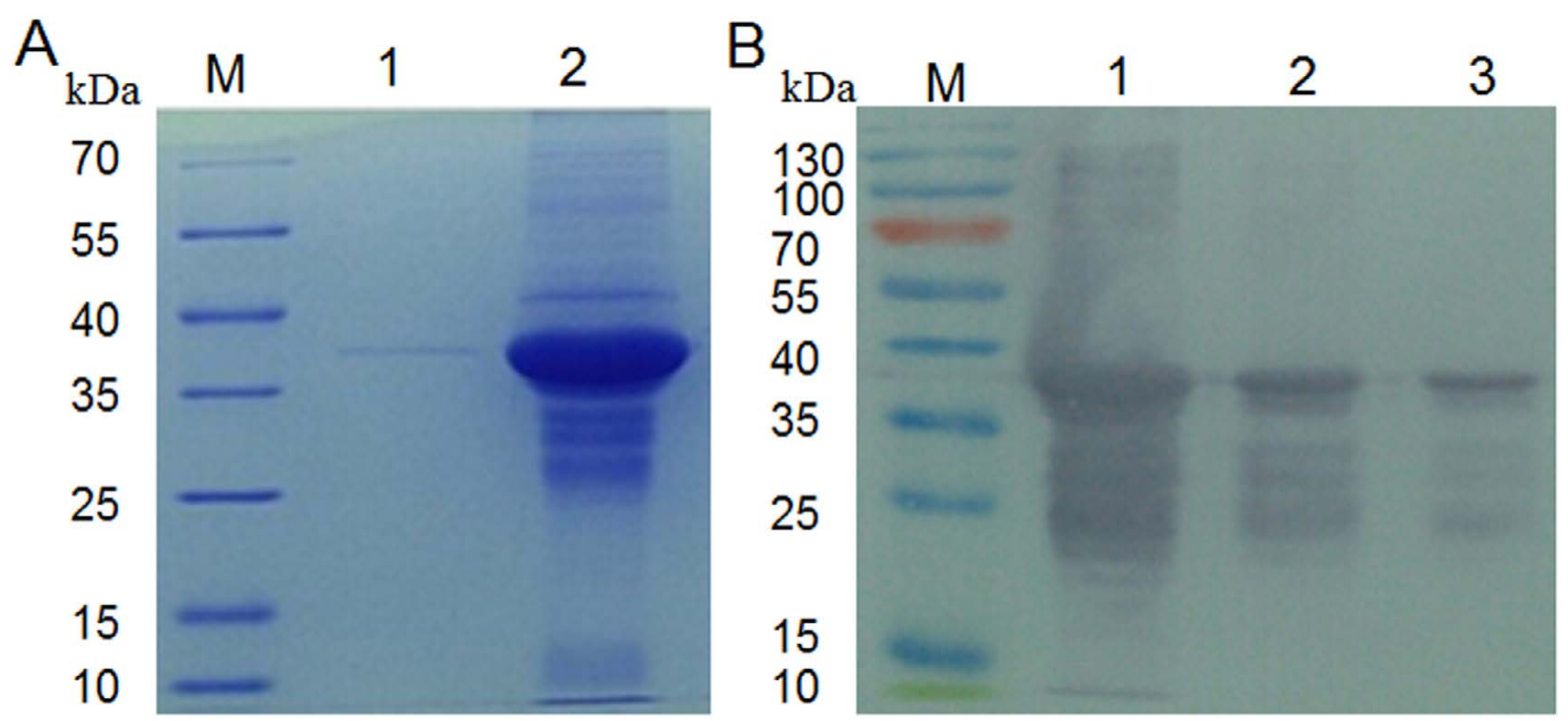

Figure 2: Prokaryotic expression, purification and identification of VP1. The vector pET-28a-VP1 was transformed into E. coli strain BL21, and GST-VP1 was expressed and purified on a GST-affinity column, and then analyzed by western blot. A. SDS-PAGE detection of unpurified (lane 1) and purified (lane 2) VP1 protein, $37 \mathrm{kDa}$ in size. B. Western blot analysis of VP1 using sera from mice challenged with EV71 (lane 1), mice immunized with expressed VP1 (lane 2), and a commercialized VP1 polyclonal antibody (lane 3).

\begin{tabular}{|c|c|c|c|c|c|c|c|}
\hline Detection & \multicolumn{5}{|c|}{ Dilution fold } \\
\hline VP1 & $1: 1$ & $1: 2$ & $1: 4$ & $1: 8$ & $1: 16$ & $1: 32$ \\
\hline Positive serum & A & A & $1: 128$ & $1: 128$ & $1: 64$ & $1: 32$ \\
\hline
\end{tabular}

Different antigen concentrations were used to sensitize $1 \%$ latex beads with an overnight reaction time (as the starting condition recommended by the manufacturer). Standard positive serum was serially diluted for reaction with the sensitized latex beads.

Table 2: Optimization of protein concentration for latex bead sensitization

\begin{tabular}{|c|c|c|c|c|c|c|c|c|c|c|c|c|c|}
\hline \multicolumn{14}{|c|}{ Sensitization time } \\
\hline \multicolumn{2}{|c|}{2} & \multicolumn{2}{|c|}{3} & \multicolumn{2}{|c|}{4} & \multicolumn{3}{|c|}{5} & 6 & \multicolumn{2}{|c|}{7} & \multicolumn{2}{|c|}{8} \\
\hline S T & LAT & $S T$ & LAT & $S T$ & LAT & ST & LAT & S T & LAT & S T & LAT & ST & LAT \\
\hline $1: 1$ & + & $1: 1$ & ++ & $1: 1$ & +++ & $1: 1$ & ++++ & $1: 1$ & ++++ & $1: 1$ & ++++ & $1: 1$ & ++++ \\
\hline $1: 2$ & $+/-$ & $1: 2$ & ++ & $1: 2$ & ++ & $1: 2$ & +++ & $1: 2$ & ++++ & $1: 2$ & ++++ & $1: 2$ & ++++ \\
\hline $1: 4$ & - & $1: 4$ & + & $1: 4$ & ++ & $1: 4$ & +++ & $1: 4$ & +++ & $1: 4$ & +++ & $1: 4$ & +++ \\
\hline $1: 8$ & - & $1: 8$ & - & $1: 8$ & ++ & $1: 8$ & ++ & $1: 8$ & +++ & $1: 8$ & +++ & $1: 8$ & +++ \\
\hline $1: 16$ & - & $1: 16$ & - & $1: 16$ & + & $1: 16$ & ++ & $1: 16$ & +++ & $1: 16$ & +++ & $1: 16$ & +++ \\
\hline $1: 32$ & - & $1: 32$ & - & $1: 32$ & - & $1: 32$ & ++ & $1: 32$ & ++ & $1: 32$ & ++ & $1: 32$ & ++ \\
\hline $1: 64$ & - & $1: 64$ & - & $1: 64$ & - & $1: 64$ & + & $1: 64$ & ++ & $1: 64$ & ++ & $1: 64$ & ++ \\
\hline $1: 128$ & - & $1: 128$ & - & $1: 128$ & - & $1: 128$ & - & $1: 128$ & + & $1: 128$ & + & $1: 128$ & + \\
\hline $1: 256$ & - & $1: 256$ & - & $1: 256$ & - & $1: 256$ & - & $1: 256$ & - & $1: 256$ & - & $1: 256$ & - \\
\hline
\end{tabular}

VP1 proteins at a 1:8 dilution were used to sensitize $1 \%$ latex beads with various reaction times $(2,3,4,5$, and 6 hours). The best reaction time was determined via a reaction with standard positive serum in serial twofold dilutions (from 1:1 to 1:256).

Table 3: Optimization of reaction time for latex bead sensitization

\begin{tabular}{|c|c|c|c|c|c|}
\hline Positive Serum & $\mathbf{0 0 1}$ & $\mathbf{0 0 2}$ & $\mathbf{0 0 3}$ & $\mathbf{0 0 4}$ & $\mathbf{0 0 5}$ \\
\hline CoxA16 & +++ & +++ & +++ & - & -+++ \\
\hline Norovirus & - & - & - & - \\
\hline ECHO-virus & - & - & - & - \\
\hline EV70 & - & - & - & - \\
\hline HAV & - & - & - & - \\
\hline
\end{tabular}

For specificity determination, six lots of theVP1-LAT were used to test standard serum positive for a wide range of enteroviruses, such as coxsackievirus A16, norovirus, enteric cytopathic human orphan (ECHO)-virus, EV70, HAV, in addition to EV71 as a positive control.

Table 4: Specificity, repeatability, and stability of the VP1-LAT 
Citation: Liu X,Qin B, He T,Chen J,Cao D, et al.(2015) Development of a Specific Latex Agglutination Test to Detect Antibodies of Enterovirus 71. Metabolomics 5: 142. doi:10.4172/2153-0769.1000142
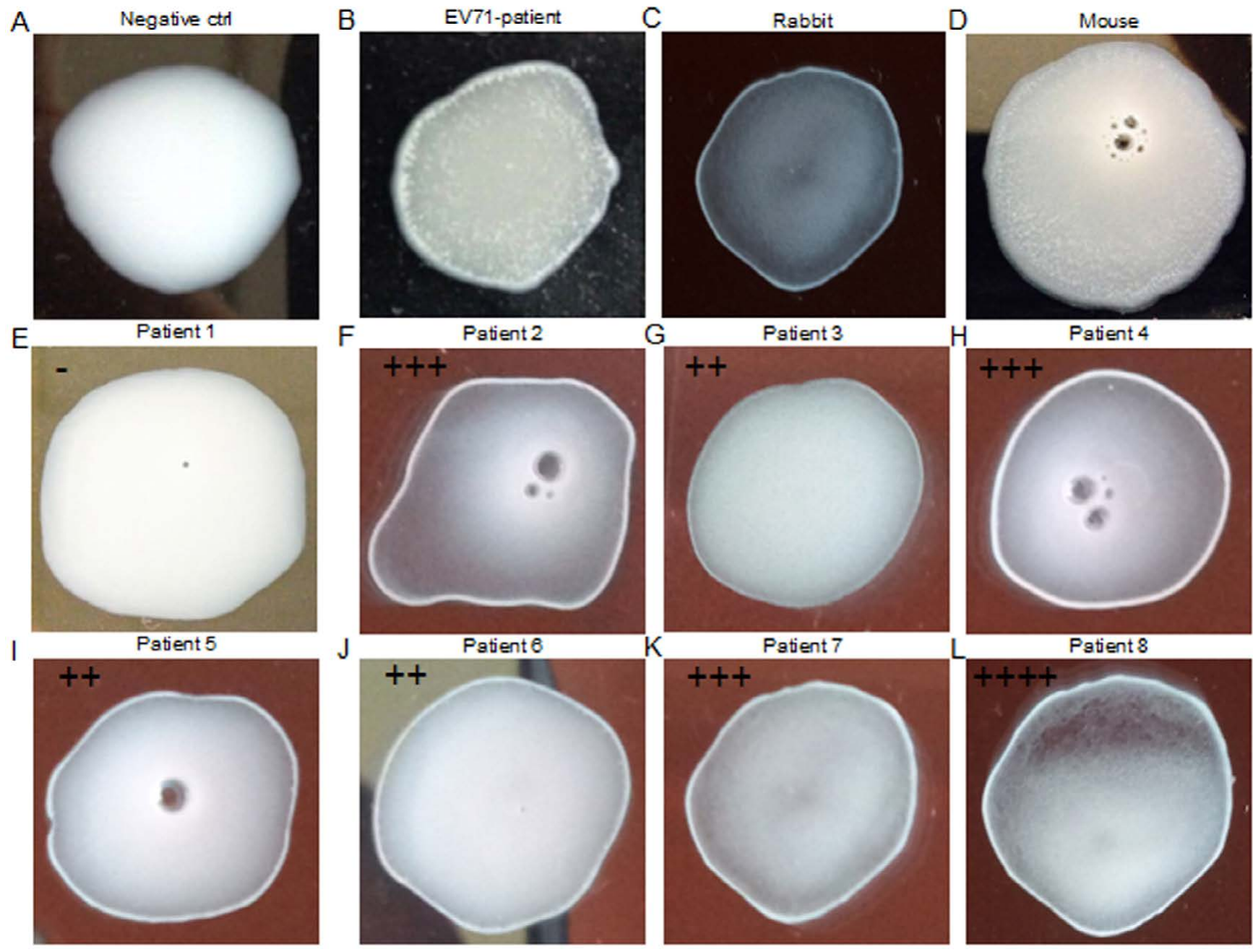

Figure 3: VP1-conjugated latex beads react well with anti-VP1 in different kinds of sera. $15 \mu$ l of sensitized latex beads were mixed and rocked with $15 \mu$ l of different kinds of sera: negative control (A), serum from an EV71-infected patient (B), serum from EV71-challenged rabbit (C), serum from an EV71-challenged mice (D), and 8 serum samples of patients (E-L).

\section{Comparison of VP1-LAT and ELISA}

The sensitivity and specificity of the LAT were evaluated by performing both the LAT and ELISA on 100 serum samples from experimentally infected mice and 608 clinical serum samples. In the 100 serum samples from experimentally infected mice, 87 were positive and 13 were negative by ELISA, whereas 84 were positive and 16 were negative by LAT, and 79 were positive by both assays and 11 were negative in both assays. According to this data, the specificity, sensitivity, and agreement ratio with the ELISA for the VP1-LAT was $90.8 \%, 84.6 \%$, and $90.0 \%$ (Table 5). In the 608 clinical serum samples, including 408 samples from children from one kindergarten in which there was a HFMD outbreak and 200 samples from healthy adults. 212 were positive and 396 were negative by ELISA, whereas 189 were positive and 419 were negative by LAT, while 184 were positive in both assays and 383 were negative in both assays. According to this data, the specificity, sensitivity, and agreement ratio with ELISA for the VP1-LAT was $86.8 \%, 96.7 \%$, and $93.3 \%$ (Table 5). For the total of 708 samples, the specificity, sensitivity, and agreement ratio with ELISA for the VP1-LAT was $88.0 \%, 96.3 \%$, and $92.8 \%$.

\section{Discussion}

HFMD, which generally affects children under the age of five, mainly caused by EV71 and coxsackie virus A16 (CVA16), syndromes caused by them are similar and indistinguishable [11]. However, EV71 infection is more serious than CVA16, and it is frequently associated with serious neurological complications and fatalities.

In China, in 2008, about 490,000 HFMD infections and 126 deaths in infants and young children were reported [1]. To date, neither an effective vaccine nor antiviral treatment is available for EV71 infection [25]. Thus, effective vaccines that could provide protection against EV71-associated HFMD or herpangina are urgently needed [6,9]. To evaluate the level of humoral immunity after vaccination, a fast detection method that is inexpensive and simple and easy to operate is required. The LAT, a proactive response to such demands, was confirmed to detect EV71-IgG from both EV71-infected children and mice and rabbits vaccinated with live or inactivated virus. Because it does not require a secondary antibody, as does ELISA, the LAT could detect anti-VP1 IgG from all kinds of sera from mice, rabbits, people, and so on. EDC is a water-soluble dehydrating agent that could 
Citation: Liu X,Qin B, He T,Chen J,Cao D, et al.(2015) Development of a Specific Latex Agglutination Test to Detect Antibodies of Enterovirus 71 Metabolomics 5: 142. doi:10.4172/2153-0769.1000142

Page 6 of 7

\section{ELISA}

\begin{tabular}{|c|c|c|c|c|c|c|}
\hline \multicolumn{4}{|c|}{100 experimental samples } & \multicolumn{3}{|c|}{608 clinical serum samples } \\
\hline & Total & Positive & Negative & Total & Positive & Negative \\
\hline \multicolumn{7}{|l|}{ LAT } \\
\hline Total & 100 & 87 & 13 & 608 & 212 & 396 \\
\hline Positive & 84 & 79 & 2 & 189 & 184 & 13 \\
\hline Negative & 16 & 5 & 11 & 419 & 36 & 383 \\
\hline
\end{tabular}

Experimental samples Sensitivity: $79 / 87=90.8 \%$; specificity: $11 / 13=84.6 \%$; agreement ratio: $(79+11) / 100=90.0 \%$.

Clinical serum samples Sensitivity: $184 / 212=86.8 \%$; specificity: $383 / 396=96.7 \%$; agreement ratio: $(184+383) / 608=93.3 \%$.

Total samples Sensitivity: $79+184 / 87+212=88.0 \%$; specificity: $(11+383) /(13+396)=96.3 \%$; agreement ratio: $(79+11+184+383) /(100+608)=92.8 \%$.

Table 5: Comparison of the LAT and ELISA in testing serum samples

revitalize the carboxyl groups of latex beads and induce the generation of amides or esters. When latex beads were first used in testing, when target proteins were connected to traditional latex beads without EDC, the binding was too weak [17,22]. In our study, EDC plays a key role as a bridge to covalently connect latex beads and target proteins, and this binding was much stronger than with traditional latex beads.

The EV71 particle consists of a naked icosahedral capsid, on which VP1 capsid protein located [26]. VP1 harbors the main neutralizing epitopes of EV71, bears the largest immune selective pressure, is a hot spot in the field of vaccine research, and was reported to carry the primary antigenic determinant of EV71. Anti-VP1 IgG is undoubtedly a logical neutralizing antibody of EV71 $[9,27]$. Hence, we developed VP1-EV71-LAT using an E. coli-expressed GST-VP1 fusion protein to sensitize latex beads. After a series of conditions were optimized, we used the LAT to detect EV71 in clinical specimens. In comparison with commercial ELISA kits, the specificity, sensitivity, and agreement ratio for a total of 708 serum samples (from both experimentally infected mice and clinical serum samples) were $86.8 \%, 96.7 \%$, and 93.3\%, respectively. The data show that the VP1-LAT exhibited high specificity, sensitivity, and agreement ratio, and could be widely used to detect anti-VP1 IgG from any kind of serum samples, as opposed to ELISA, especially to evaluate the effect of vaccination fast when an inactivated vaccine against EV71 is available.

The LAT established in our study shows satisfactory specificity to selectively differentiate antibodies against EV71 from those of other enteroviruses, and it also exhibits outstanding within-lot and lot-to-lot repeatability, and stability. The VP1-LAT is suitable for the rapid and simple serologic detection of antibodies from humans and a variety of target animals against EV71-VP1, or virons. The VP1-LAT has broad clinical application prospects.

The degree of aggregation, as determined by the naked eye, was slightly subjective. As a matter of fact, latex beads are milk-like before any reaction, and a clear solution and aggregated particles will appear if they react with positive samples. The translucency of the reaction system and particle size was closely correlated to the degree of positive reaction. Furthermore, we will try to improve the method of LAT assessment in a future study by detecting the size and amount of aggregated particles or the translucency of the reaction mixture.

\section{Acknowledgments}

We are grateful to the patients for providing the serum samples. This work was supported by Science Technology Bureau of Shaoxing, Zhejiang province of China (2013B70063 and 2012B70063) and Zhejiang Medical Technology \& Education (2013KYA208).

\section{References}

1. Zhu F, Xu W, Xia J, Liang Z, Liu Y, et al. (2014) Efficacy, safety, and immunogenicity of an enterovirus 71 vaccine in China. J Med 370: 818-828.
2. Blomberg J, Lycke E, Ahlfors K, Johnsson T, Wolontis S, et al. (1974) Letter: New enterovirus type associated with epidemic of aseptic meningitis and-or hand, foot, and mouth disease. Lancet 2: 112.

3. Ho M, Chen ER, Hsu KH, Twu SJ, Chen KT, et al. (1999) An epidemic of enterovirus 71 infection in Taiwan. Taiwan Enterovirus Epidemic Working Group. J Med 341: 929-935.

4. Li YP, Liang ZL, Xia JL, Wu JY, Wang L, et al. (2014) Immunogenicity, safety, and immune persistence of a novel inactivated human enterovirus 71 vaccine: a phase II, Randomized, double-blind, placebo-controlled. Trial J Infect Dis 209 46-55.

5. Wang SM, Lei HY, Huang KJ, Wu JM, Wang JR, et al. (2003) Pathogenesis of enterovirus 71 brainstem encephalitis in pediatric patients: roles of cytokines and cellular immune activation in patients with pulmonary edema. $\mathrm{J}$ Infect Dis 188: $564-570$.

6. Zhu FC, Meng FY, Li JX, Li XL, Mao QY, et al. (2013) Efficacy, safety, and immunology of an inactivated alum-adjuvant enterovirus 71 vaccine in children in China: a multicentre, randomised, double-blind, placebo-controlled, phase 3 trial. Lancet 381: 2024-2032.

7. McMinn P, Stratov I, Nagarajan L, Davis S (2001) Neurological manifestations of enterovirus 71 infection in children during an outbreak of hand, foot, and mouth disease in Western Australia. Clin Infect Dis 32: 236-242.

8. Li W, Yi L, Su J, Lu J, Ke C, et al. (2013) Seroprevalence of human enterovirus 71 and coxsackievirus A16 in Guangdong, China, in pre- and post-2010 HFMD epidemic period. PLoS One 8: e80515.

9. Chung YC, Ho MS, Wu JC, Chen WJ, Huang JH, et al. (2008) Immunization with virus-like particles of enterovirus 71 elicits potent immune responses and protects mice against lethal challenge. Vaccine 26: 1855-1862.

10. Wang RY, Kuo RL, Ma WC, Huang HI, Yu JS, et al. (2013) Heat shock protein90-beta facilitates enterovirus 71 viral particles assembly. Virology 443: 236247.

11. Lu J, Yi L, Zhao J, Yu J, Chen Y, et al. (2012) Enterovirus 71 disrupts interferon signaling by reducing the level of interferon receptor 1. J Virol 86: 3767-3776.

12. He F, Kiener TK, Lim XF, Tan Y, Raj KV, et al. (2013) Development of a sensitive and specific epitope-blocking ELISA for universal detection of antibodies to human enterovirus 71 strains. PLoS One 8:e55517.

13. Ma S, Mao Q, Liang Z, Zhang C, Yang W, et al. (2013) Development of a sandwich ELISA for the quantification of enterovirus 71. Cytotechnology.

14. Tan EL, Yong LL, Quak SH, Yeo WC, Chow VT, et al. (2008) Rapid detection of enterovirus 71 by real-time TaqMan RT-PCR. J Clin Virol 42: 203-206.

15. Xiao XL, He YQ, Yu YG, Yang H, Chen G, et. al (2009) Simultaneous detection of human enterovirus 71 and coxsackievirus A16 in clinical specimens by multiplex real-time PCR with an internal amplification control. Arch Virol 154:121-125.

16. Yu XL, Tu CC, Xu XR, Zhang ML, Chen YX, et al. (2003) [Site-directed mutagensis of the major antigen E2 gene of CSFV, its high level expression in Escherichia coli and the immunonicity of recombinant E2 protein]. Sheng $\mathrm{Wu}$ Gong Cheng Xue Bao 19: 439-443.

17. Inzana TJ (1995) Simplified procedure for preparation of sensitized latex particles to detect capsular polysaccharides: application to typing and diagnosis of Actinobacillus pleuropneumoniae. J Clin Microbiol 33: 2297-2303. 
Citation: Liu X,Qin B, He T,Chen J,Cao D, et al.(2015) Development of a Specific Latex Agglutination Test to Detect Antibodies of Enterovirus 71. Metabolomics 5: 142. doi:10.4172/2153-0769.1000142

18. Yu Z, Jin M, Xu X, Zhang R, Zhou H, et al. (2006) Development of a specific latex agglutination test based on a recombinant hemagglutinin protein to detect antibodies to H5 avian influenza viruses. Avian Dis 50:264-268.

19. Yip CC, Lau SK, Lo JY, Chan KH, Woo PC, et al. (2013) Genetic characterization of EV71 isolates from 2004 to 2010 reveals predominance and persistent circulation of the newly proposed genotype $D$ and recent emergence of a distinct lineage of subgenotype C2 in Hong Kong. Virol J 10: 222.

20. Zhu J, Luo Z, Wang J, Xu Z, Chen H, et al. (2013) Phylogenetic analysis of Enterovirus 71 circulating in Beijing, China from 2007 to 2009. PLoS One 8: e56318.

21. Qin B, Budeus B, Cao L, Wu C, Wang Y, et al. (2013) The amino acid substitutions rtP177G and rtF249A in the reverse transcriptase domain of hepatitis B virus polymerase reduce the susceptibility to tenofovir. Antiviral Res 97 93-100.

22. Ortega-Vinuesa JL, Bastos-González D, Hidalgo-Álvarez R (1996) Effect of Storage Time on the Immunoreactivity of IgG Physically Adsorbed or Chemically Bound to Latex Beads. J Colloid Interface Sci 184: 331-334.
23. Qin B, Wan X, Hu Q, Yu Z, Chao Y, et al. (2007) Development and application of a latex agglutination test based on the MPB70 protein of Mycobacterium bovis. Chinese Journal of Preventive Veterinary Medicine 29: 5.

24. Brown BA, Kilpatrick DR, Oberste MS, Pallansch MA (2000) Serotype-specific identification of enterovirus 71 by PCR. J Clin Virol 16: 107-112.

25. Fan X, Jiang J, Liu Y, Huang X, Wang P, et al. (2013) Detection of human enterovirus 71 and Coxsackievirus A16 in an outbreak of hand, foot, and mouth disease in Henan Province, China in 2009. Virus Genes 46: 1-9.

26. Qing J, Wang Y, Sun Y, Huang J, Yan W, et al. (2014) Cyclophilin A associates with enterovirus-71 virus capsid and plays an essential role in viral infection as an uncoating regulator. PLoS Pathog 10: e1004422.

27. Zhang H, An D, Liu W, Mao Q, Jin J, et al. (2014) Analysis of cross-reactive neutralizing antibodies in human HFMD serum with an EV71 pseudovirusbased assay. PLoS One 9: e100545. 